\title{
Decoding Schemes for FBMC with Single-Delay STTC
}

\author{
Chrislin Lélé and Didier Le Ruyet (EURASIP Member) \\ Electronics and Communications Laboratory, Conservatoire National Des Arts Et Métiers (CNAM), 75141 Paris, France
}

Correspondence should be addressed to Didier Le Ruyet, didier.le_ruyet@cnam.fr

Received 5 June 2009; Accepted 28 December 2009

Academic Editor: Markku Renfors

Copyright ( 2010 C. Lélé and D. Le Ruyet. This is an open access article distributed under the Creative Commons Attribution License, which permits unrestricted use, distribution, and reproduction in any medium, provided the original work is properly cited.

\begin{abstract}
Orthogonally multiplexed Quadrature Amplitude Modulation (OQAM) with Filter-Bank-based MultiCarrier modulation (FBMC) is a multicarrier modulation scheme that can be considered an alternative to the conventional orthogonal frequency division multiplexing (OFDM) with cyclic prefix (CP) for transmission over multipath fading channels. However, as OQAM-based FBMC is based on real orthogonality, transmission over a complex-valued channel makes the decoding process more challenging compared to CP-OFDM case. Moreover, if we apply Multiple Input Multiple Output (MIMO) techniques to OQAM-based FBMC, the decoding schemes are different from the ones used in CP-OFDM. In this paper, we consider the combination of OQAM-based FBMC with single-delay Space-Time Trellis Coding (STTC). We extend the decoding process presented earlier in the case of $N_{t}=2$ transmit antennas to greater values of $N_{t}$. Then, for $N_{t} \geq 2$, we make an analysis of the theoretical and simulation performance of $\mathrm{ML}$ and Viterbi decoding. Finally, to improve the performance of this method, we suggest an iterative decoding method. We show that the OQAM-based FBMC iterative decoding scheme can slightly outperform CP-OFDM.
\end{abstract}

\section{Introduction}

Orthogonal Frequency Division Multiplexing (OFDM) is an efficient Multicarrier Modulation (MCM) capable of fighting against multipath fading channels. Its robustness to multipath propagation effects comes from the insertion of a CP and is, therefore, obtained at the price of a reduced spectral efficiency. Furthermore, the rectangular shape of OFDM symbols leads to a $\sin (x) / x$ frequency spectrum. Studies have been conducted in order to find better MCM schemes with respect to the frequency and/or time-frequency localization criteria.

As suggested in [1-3], OFDM/OQAM also called as OQAM-based Filter Bank Multicarrier (FBMC) is an MCM scheme which may be the appropriate alternative. In OFDM/OQAM each subcarrier is modulated with Offset Quadrature Amplitude Modulation (OQAM). This principle has been introduced in $[4,5]$, but it is only recently [1] that FBMC has been presented as a viable alternative to OFDM. Compared to OFDM that transmits complex-valued symbols at a given symbol rate, OQAM-based FBMC transmits realvalued symbols at twice this symbol rate. Therefore, a similar spectral efficiency is achieved by both systems. In practice, OQAM-based FBMC may provide a higher useful bit rate since it operates without the addition of a CP. Furthermore, with a pulse shaping that can be optimized according to given channel characteristics, its performance can be improved. However, all the interesting features of OQAM-based FBMC come at the price of a relaxation of the orthogonality conditions that only hold in the real field. At the receive side the data is carried only by the real component of the signal (assuming a 0 or $\pi / 2$ phase modulation term). Thus, the imaginary part appears as an interference term. This interference term is a source of problem in the presence of the complex-valued channel as it destroys the real orthogonality. Therefore, when combining OQAMbased FBMC with MIMO technique such as Space-Time Block Codes (STBC) or Space-Time Trellis Coding (STTC) $[6,7]$, the decoding process cannot be done in the same way as with CP-OFDM modulation. In the case of a single delay STTC chain with 2 transmit and 1 receive antennas, refrence [8] proposed a simple preprocessing to cancel this imaginary interference component. In this paper, we extend the proposed method in [8] to $N_{t}$ transmit antennas and introduce an iterative decoding method. In Section 2, we give a short description of the discrete-time OQAM modulation. 
Then, in Section 3, we provide an overview of the STTC single delay detection. In Section 4.1, we provide a theoretical performance analysis of ML and Viterbi decoding. Section 5 is devoted to the iterative decoding method in order to improve the performance of the previous decoding method. Simulation results are presented in Section 6. Conclusions and perspectives are given in Section 7. In the rest of the paper, FBMC will be used to denote OQAM-based FBMC.

\section{The FBMC Modulation}

Using the baseband discrete-time model, we can write at the transmit antenna $i$ the OQAM-based FBMC signal as follows [1]:

$$
s^{i}[m]=\sum_{k=0}^{M-1} \sum_{n \in \mathbf{Z}} d_{k, n, i} \underbrace{g[m-n N] e^{j(2 \pi / M) k(m-D / 2)} e^{j \phi_{k, n}}}_{g_{k, n}[m]},
$$

where $M=2 N$ is the even number of subcarriers, $F_{0}=$ $1 / T_{0}=1 / 2 \tau_{0}$ is the subcarrier spacing, $\phi_{k, n}$ is an additional phase term, $g$ is the pulse shape, and $D$ is the delay parameter associated to the length of the pulse shape. The transmitted symbols $d_{k, n, i}$ are real-valued data transmitted by antenna $i$. They are obtained from a $2^{2 K}$-QAM constellation, taking the real and imaginary parts of these complex-valued symbols of duration $T_{0}=2 \tau_{0}$, where $\tau_{0}$ denotes the time offset between the two parts $[1-3,9]$. For a given subcarrier $k$ and symbol time index $n$, the real and imaginary parts are driven by the phase term $\phi_{k, n}$ given by

$$
\phi_{k, n}=\phi_{0}+\frac{\pi}{2}(n+k)(\bmod \pi)
$$

where $\phi_{0}$ can be arbitrarily chosen. Here, we set $\phi_{0}=0$ and $g$ is assumed to be real valued.

Assuming a distortion-free channel, a perfect reconstruction of real symbols is obtained owing to the following real orthogonality condition:

$$
\mathfrak{R}\left\{\left\langle g_{k, n} \mid g_{p, q}\right\rangle\right\}=\mathfrak{R}\left\{\sum_{m=-\infty}^{\infty} g_{k, n}[m] g_{p, q}^{*}[m]\right\}=\delta_{k, p} \delta_{n, q},
$$

where $\delta_{k, p}=1$ if $k=p$ and $\delta_{n, q}=0$ if $n \neq q$. However, in practice for transmission over a realistic channel, the orthogonality property is lost, leading to intersymbol and intercarrier interferences. It has been shown in previous studies [8] that, when combining FBMC with single delay STTC in presence of 2 transmit and one receive antennas, specific processing should be done in order to remove the interference terms. In this paper, we will extend this method for $N_{t} \geq 2$ antennas.

\section{Single-Delay STTC in FBMC with $N_{t}$ Transmit Antennas}

3.1. Transmission Model. Let us first assume that only the $i$ th antenna is transmitting. At the receiver side, the demodulated signal $y_{k, n}$ at the frequency $k$ and time instant $n\left(n \tau_{0}\right)$ can be written as

$$
y_{k, n}=H_{k, n, i} d_{k, n, i}+j I_{k, n, i}+v_{k, n},
$$

where

(i) $H_{k, n, i}$ is the channel coefficient between transmit antenna $i$ and the receiver, at subcarrier $k$ and time instant $n$,

(ii) $v_{k, n}$ is the noise component at subcarrier $k$ and time instant $n$,

(iii)

$$
I_{k, n, i}=(-j) \sum_{\left(k^{\prime}, n^{\prime}\right) \neq(k, n)} H_{k^{\prime}, n^{\prime}, i} d_{k^{\prime}, n^{\prime}, i} \sum_{m=-\infty}^{\infty} g_{k, n}[m] g_{k^{\prime}, n^{\prime}}^{*}[m] .
$$

We assume that we have a prototype filter well localized in time and frequency. This implies that in the previous equation the main contribution comes from the closest neighborhood, that is, $g_{k, n}[m] g_{k^{\prime}, n^{\prime}}^{*}[m]$ takes a significant value only for $\left|k-k^{\prime}\right| \leq 1$ and $\left|n-n^{\prime}\right| \leq 1$. Moreover, if we assume that the channel is constant over a set of at least three consecutive subcarriers and a set of at least three consecutive time indexes, then we can rewrite the previous expression as in $[10]$ :

$$
I_{k, n, i} \approx H_{k, n, i}(-j) \underbrace{}_{u_{k, n, i} \sum_{\left(k^{\prime}, n^{\prime}\right) \neq(k, n)} d_{k^{\prime}, n^{\prime}, i} \sum_{m=-\infty}^{\infty} g_{k, n}[m] g_{k^{\prime}, n^{\prime}}^{*}[m]} .
$$

Thus, the demodulated signal can be approximated by

$$
y_{k, n} \approx H_{k, n, i}\left(d_{k, n, i}+j u_{k, n, i}\right)+v_{k, n} .
$$

Throughout the remainder of the paper, we will consider (7) as the expression of the signal at the output of the demodulator.

3.2. Problem Statement. Let us consider the single delay STTC scheme with $N_{t}$ antennas as shown in Figure 1. The real data to be transmitted is modulated by an FBMC modulator and transmitted by the first antenna. The same stream of data is delayed by $2 n_{i}$ real data before being modulated by FBMC modulator and transmitted by the $n_{i}$ th antenna. The delay $2 n_{i}$ is chosen to have the same delay as with a CP-OFDM system although a delay of $n_{i}$ could also be chosen. We denote by $a_{k, n}$ the real data from the main stream of data at frequency $k$ and time index $n$. Thus, at a given subcarrier $k$ the transmission is given at antenna $i$ by $d_{k, n, i}=a_{k, n-2 i}$. At the receiver side, the demodulated signal can be written as

$$
y_{k, n}=\sum_{i=0}^{N_{t}-1} H_{k, n, i}\left(d_{k, n, i}+j u_{k, n, i}\right)+v_{k, n},
$$

where $v_{k, n}$ is the noise component at the subcarrier $k$ and time instant $n$. As the same stream of data is transmitted over the $N_{t}$ antennas, we have $u_{k, n, i}=u_{k, n-2 i, 0}=b_{k, n-2 i}$. In the 
remainder of the paper, we will assume a channel constant over time, that is, $\left(H_{k, n, i}=H_{k, i}\right)$; we get

$$
y_{k, n}=\sum_{i=0}^{N_{t}-1} H_{k, i} \underbrace{\left(a_{k, n-2 i}+j b_{k, n-2 i}\right)}_{x_{k, n-2 i}}+v_{k, n} .
$$

The problem is to recover from $y_{k, n}$ the data $a_{k, n}$. The presence of the term $b_{k, n-2 i}$ makes the decoding process from $y_{k, n}$ difficult. Some processing should be carried out in order to recover the real data.

\section{Interference Cancelation Method}

4.1. Cancelation Procedure. For the case $N_{t}=2$, it has been shown in [8] that if we define $z_{k, n+2}$ as

$$
z_{k, n+2}=H_{k, 1}^{*} y_{k, n}+H_{k, 0}^{*} y_{k, n+2}
$$

then we have

$$
\begin{aligned}
\mathfrak{R}\left\{z_{k, n+2}\right\}= & \mathfrak{R}\left\{H_{k, 1}^{*} y_{k, n}+H_{k, 0}^{*} y_{k, n+2}\right\} \\
= & \left|H_{k, 1}\right|^{2} a_{k, n-2}+2 \mathfrak{R}\left\{H_{k, 1}^{*} H_{k, 0}\right\} a_{k, n} \\
& +\left|H_{k, 0}\right|^{2} a_{k, n+2}+w_{k, n+2},
\end{aligned}
$$

with $w_{k, n+2}=\mathfrak{R}\left\{H_{k, 1}^{*} v_{k, n}+H_{k, 0}^{*} v_{k, n+2}\right\}$. Let $2 L_{f}$ denotes the frame length, for $e \in\{0,1\}$. If we denote by

$$
\begin{aligned}
& \underline{t}_{e}=\left[\begin{array}{lllll}
\mathfrak{R}\left\{z_{k, e}\right\} & \mathfrak{R}\left\{z_{k, e+2}\right\} & \cdots & \mathfrak{R}\left\{z_{k, e+2\left(L_{f}-1\right)}\right\}
\end{array}\right]^{T}, \\
& \underline{a}_{e}=\left[\begin{array}{llll}
a_{k, e} & a_{k, e+2} & \cdots & a_{k, e+2\left(L_{f}-1\right)}
\end{array}\right]^{T}, \\
& \underline{w}_{e}=\left[\begin{array}{llll}
w_{k, e} & w_{k, e+2} & \cdots & w_{k, e+2\left(L_{f}-1\right)}
\end{array}\right]^{T} \\
& \underbrace{\underline{G}_{2}}_{\left.\begin{array}{ccccc}
\left|H_{k, 0}\right|^{2} & 0 & \cdots & \cdots & 0 \\
2 \Re\left\{H_{k, 0} H_{k, 1}^{*}\right\} & \left|H_{k, 0}\right|^{2} & \ddots & \cdots & \vdots \\
\left|H_{k, 1}\right|^{2} & 2 \Re\left\{H_{k, 0} H_{k, 1}^{*}\right\} & \left|H_{k, 0}\right|^{2} & 0 & \vdots \\
0 & \ddots & \ddots & \ddots & \vdots \\
\vdots & \ddots & \ddots & \ddots & 0 \\
0 & \cdots & \left|H_{k, 1}\right|^{2} & 2 \Re\left\{H_{k, 0} H_{k, 1}^{*}\right\} & \left|H_{k, 0}\right|^{2}
\end{array}\right]}
\end{aligned}
$$

$\left((\cdot)^{T}\right.$ denotes the transpose operation and $(\cdot)^{H}$ the transpose conjugate one) then we have

$$
\underline{t}_{e}=\underline{G}_{2} \underline{a}_{e}+\underline{w}_{e}
$$

In this last equation, the imaginary interference term is canceled. Thus the decoding process can be easily carried out by using either Maximum Likelihood (ML) decoding, Viterbi decoding, or linear equalization such as Zero Forcing (ZF) or Minimum Mean Square Error (MMSE) decoding. More generally with $N_{t} \geq 2$, let us note and compute

$$
\begin{aligned}
z_{k, n+2 N_{t}-2}= & \sum_{p=0}^{N_{t}-1} H_{k, N_{t}-1-p}^{*} y_{k, n+2 p} \\
= & \sum_{p=0}^{N_{t}-1} \sum_{i=0}^{N_{t}-1} H_{k, N_{t}-1-p}^{*} H_{k, i} x_{k, n+2 p-2 i} \\
& +\underbrace{\sum_{p=0}^{N_{t}-1} H_{k, N_{t}-1-p}^{*} v_{k, n+2 p}}_{n_{k, n+2 N_{t}-2}}
\end{aligned}
$$

$$
=\underbrace{\sum_{i=1}^{N_{t}-1} \sum_{p=0}^{i-1} H_{k, N_{t}-1-p}^{*} H_{k, i} x_{k, n+2 p-2 i}}_{B_{k, n}}
$$$$
+\underbrace{\sum_{i=0}^{N_{t}-1} \sum_{p=i} H_{k, N_{t}-1-p}^{*} H_{k, i} x_{k, n+2 p-2 i}}_{A_{k, n}}
$$$$
+\underbrace{\sum_{i=0}^{N_{t}-1} \sum_{p=i+1}^{N_{t}-1} H_{k, N_{t}-1-p}^{*} H_{k, i} x_{k, n+2 p-2 i}}_{C_{k, n}}
$$$$
+\sum_{p=0}^{N_{t}-1} H_{k, N_{t}-1-p}^{*} v_{k, n+2 p} .
$$

Moreover $A_{k, n}$ is given by

$$
A_{k, n}=x_{k, n} \mu_{k}
$$


and details for this equation are given in Appendix A.1. The expression of $B_{k, n}$ is given by

$$
B_{k, n}=\sum_{q=1}^{N_{t}-1} x_{k, n-2 q} \gamma_{q},
$$

where $\gamma_{q}$ are real-valued quantities which depend only on the channel coefficients as shown in Appendix A.2. The expression of $C_{k, n}$ is given by

$$
C_{k, n}=\sum_{q=1}^{N_{t}-1} x_{k, n+2 q} \beta_{q},
$$

where $\beta_{q}$ are real-valued quantities which depend only on the channel coefficients as shown in Appendix A.3. Therefore,

$$
\begin{aligned}
z_{k, n+2 N_{t}-2}= & \sum_{q=1}^{N_{t}-1} \gamma_{q} x_{k, n-2 q}+\mu_{k} x_{k, n}+\sum_{q=1}^{N_{t}-1} \beta_{q} x_{k, n+2 q} \\
& +\sum_{p=0}^{N_{t}-1} H_{k, N_{t}-1-p}^{*} v_{k, n+2 p} .
\end{aligned}
$$

Thus, by noting that $t_{k, n+2 N_{t}-2}^{(1)}=\mathfrak{R}\left\{z_{k, n+2 N_{t}-2}\right\}$, we have

$$
\begin{aligned}
t_{k, n+2 N_{t}-2}^{(1)}= & \sum_{q=1}^{N_{t}-1} \gamma_{q} a_{k, n-2 q}+\mu_{k} a_{k, n}+\sum_{q=1}^{N_{t}-1} \beta_{q} a_{k, n+2 q} \\
& +\underbrace{\Re\left\{\sum_{p=0}^{N_{t}-1} H_{k, N_{t}-1-p}^{*} v_{k, n+2 p}\right\}}_{w_{k, n+2 N_{t}-2}} .
\end{aligned}
$$

For $e \in\{0,1\}$, we note $\underline{t}_{e}=\left[\begin{array}{llll}t_{k, e} & t_{k, e+2} & \cdots & t_{k, e+2\left(L_{f}-1\right)}\end{array}\right]^{T}$, $\underline{w}_{e}=\left[\begin{array}{lllll}\mathfrak{R}\left\{w_{k, e}\right\} & \Re\left\{w_{k, e+2}\right\} & \ldots & \Re\left\{w_{k, e+2\left(L_{f}-1\right)}\right.\end{array}\right]^{T}$, and

$G_{N_{t}}$

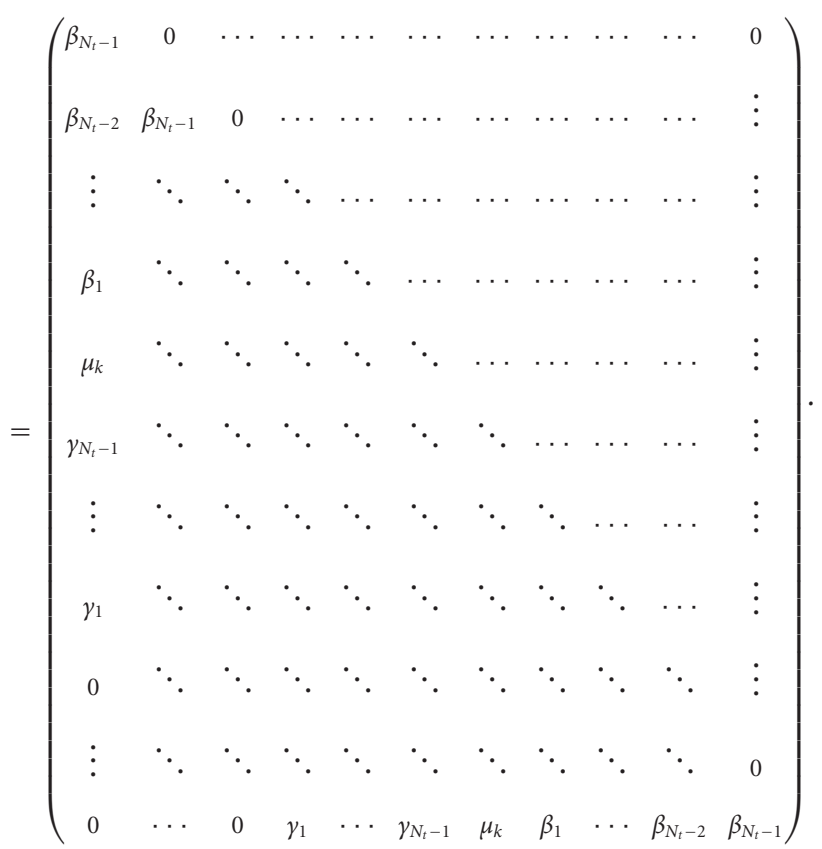

We have:

$$
\underline{t}_{e}=\underline{G}_{N_{t}} \underline{a}_{e}+\underline{w}_{e}
$$

There is no imaginary interference in (21) and consequently Maximum Likelihood (ML) [11] or linear equalizers can be used to estimate $a_{k, n}$.

The computation of $z_{k, n}$ from $y_{k, n}$ according to (14) is referred to as Preprocessing1 as shown in Figure 2. We will now provide a theoretical performance analysis of this scheme.

4.2. A Theoretical Performance Analysis. Let us consider that the noise $v_{k, n}$ is an AWGN noise with $E\left\{\left|v_{k, n}\right|^{2}\right\}=N_{0}$. It is worth noticing that $\mathfrak{R}\left\{w_{k, n}\right\}$ is Gaussian noise as it is the result of the real part of a linear transformation of Gaussian noise. However this noise is colored. For example, when $N_{T}=2$, we have

(i) $E\left\{w_{k, n} w_{k, n+2}^{*}\right\}=E\left\{w_{k, n+2} w_{k, n}^{*}\right\}=N_{0}\left(\left|H_{k, 0}\right|^{2}+\right.$ $\left.\left|H_{k, 1}\right|^{2}\right) \Re\left\{\left(H_{k, 0}\right)^{*} H_{k, 1}\right\} / 2$,

(ii) $E\left\{w_{k, n} w_{k, n}^{*}\right\}=N_{0}\left(\left|H_{k, 0}\right|^{2}+\left|H_{k, 1}\right|^{2}\right) / 2=U_{0} / 2$,

(iii) for $q \neq\{0,1\}, E\left\{w_{k, n} w_{k, n+2 q}^{*}\right\}=0$.

Let us recall that if the noise was white the ML performance would have been obtained by the Viterbi decoder. Therefore, the performance of Viterbi decoding in this present case is suboptimal. In [12] the authors evaluate the loss of performance of Viterbi decoding in presence of correlated noise. The optimal performance using an ML decoding is very complex to implement since it requires an exhaustive search over all the possible transmitted sequences. Another alternative could be to perform a whitening followed by a Viterbi decoding. However, such Viterbi decoding will be more complex since the whitening will increase the number of states. Indeed, the noise $\underline{w}_{e}$ is colored with a correlation matrix $\underline{R}$. Since $\underline{R}$ is a positive Hermitian matrix, its eigenvalues are real and positive. We have

$$
\underline{R}=\underline{Q} \underbrace{\left[\begin{array}{cccc}
\lambda_{0} & 0 & \cdots & 0 \\
0 & \ddots & \ddots & \vdots \\
\vdots & \ddots & \ddots & 0 \\
0 & \cdots & 0 & \lambda_{L_{f}-1}
\end{array}\right]}_{\underline{\Lambda}}
$$

with $\underline{Q}$ being a unitary matrix, that is, $\underline{Q Q}{ }^{H}=\underline{I_{L_{f}}}$. We denote

$$
\underline{\Lambda}^{1 / 2}=\left[\begin{array}{cccc}
\lambda_{0}^{1 / 2} & 0 & \cdots & 0 \\
0 & \ddots & \ddots & \vdots \\
\vdots & \ddots & \ddots & 0 \\
0 & \cdots & 0 & \lambda_{L_{f}-1}^{1 / 2}
\end{array}\right] .
$$




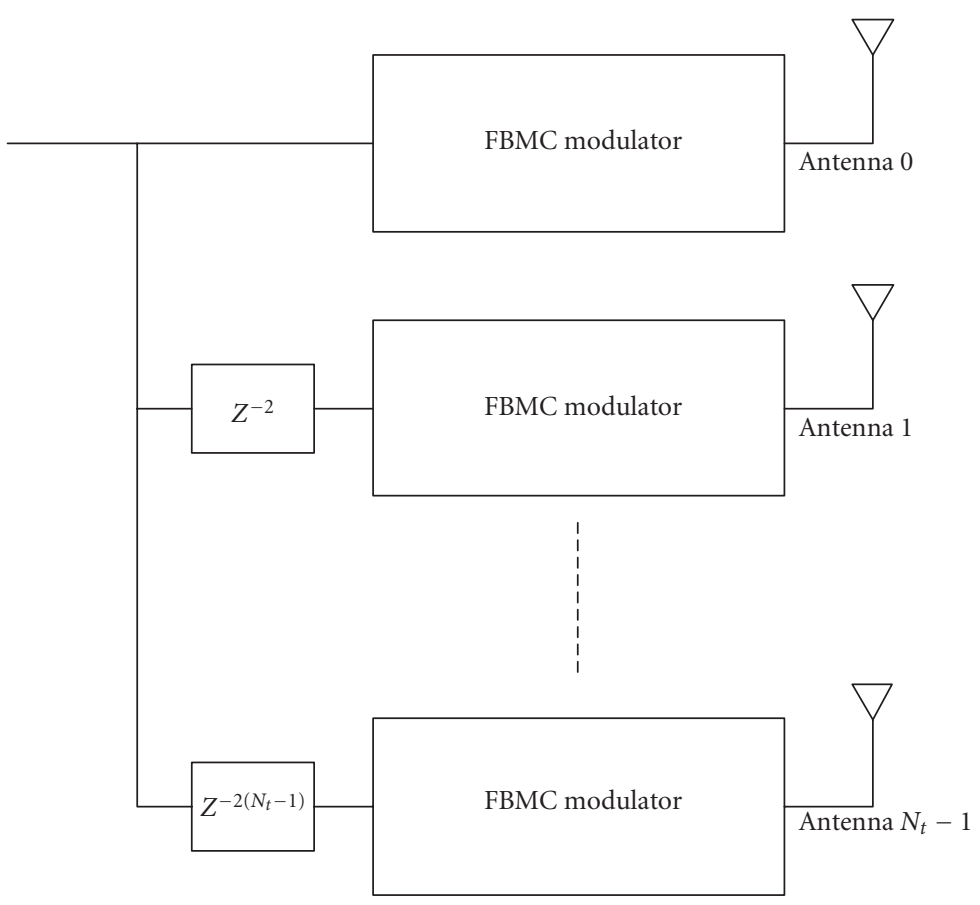

Figure 1: FBMC Single-delay STTC transmitter.

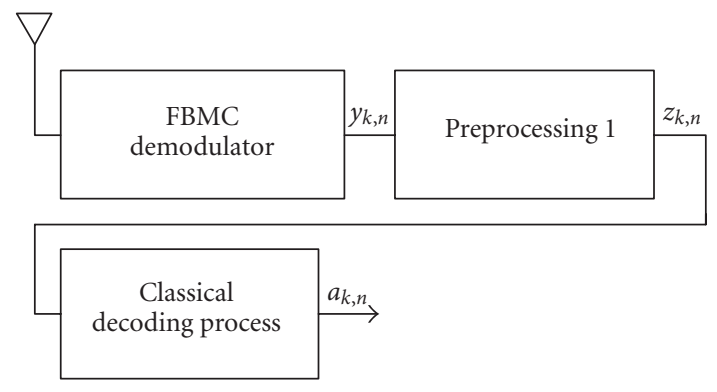

FIGURE 2: FBMC Single-delay STTC receiver.

Therefore, the whitening process can be done by computing $\underline{y}_{e}=\underline{\Lambda}^{-1 / 2} \underline{Q}^{H} \underline{z}_{e}=\underbrace{\underline{\Lambda}^{-1 / 2} \underline{Q}^{H} \underline{G}_{2}}_{\underline{\Lambda}} \underline{a}_{e}+\underbrace{\underline{\Lambda}^{-1 / 2} \underline{Q}^{H} \underline{w}_{e}}_{\underline{\mu}_{e}}=\underline{H}_{e}+\underline{\mu}_{e}$.

It can easily be proved that $\mu_{e}$ is AWGN. As we will see in the simulation results section, the presence of the colored noise will lead to a degradation of performance. Let us now present an iterative decoding approach which should improve the performance compared to that of the previous decoding strategy.

\section{Iterative Method}

5.1. Iterative Procedure. In this section we propose an iterative decoding procedure for FBMC single-delay STTC decoding. At the output of the Preprocessing1 block (see Figure 3), we can perform a decoding procedure
(ML, Viterbi, or linear decoding) to derive an estimate value $\hat{a}_{k, n}^{(1)}$ of $a_{k, n}$. From (6) and using this estimate $\hat{a}_{k, n}^{(1)}$, we can compute an estimate $\hat{u}_{k, n}^{(1)}$ of $u_{k, n}$ by

$$
\hat{u}_{k, n}^{(1)}=\sum_{(p, q) \neq(0,0)} \hat{a}_{k+p, n+q}^{(1)} \underbrace{\sum_{m=-\infty}^{\infty} g_{k, n}[m] g_{k+p, n+q}[m]}_{\gamma_{p, q}} .
$$

It is worth noticing that for a well-localized prototype filter in time and frequency domain it is enough to consider the previous sum only for $p, q \in\{1,-1\}$, that is,

$$
\hat{u}_{k, n}^{(1)} \approx \sum_{|p|=1,|q|=1} \hat{a}_{k+p, n+q}^{(1)} \gamma_{p, q} .
$$

This approximation is justified in [10]. $\gamma_{p, q}$ can be computed off-line since the prototype filter response is known. Then in (9) we can remove the contribution of the $u_{k, n}$ components by computing

$$
\begin{aligned}
y_{k, n}^{(2)}= & y_{k, n}-\sum_{i=0}^{N_{t}-1} H_{k, i} \hat{u}_{k, n-2 i}^{(1)}=\sum_{i=0}^{N_{t}-1} H_{k, i} a_{k, n-2 i} \\
& +\sum_{i=0}^{N_{t}-1} j H_{k, i}\left(u_{k, n-2 i}-\hat{u}_{k, n-2 i}^{(1)}\right)+v_{k, n} .
\end{aligned}
$$

If we assume a perfect cancelation of the $u_{k, n}$ terms, that is, $u_{k, n}=\widehat{u}_{k, n}^{(1)}$, then we have

$$
y_{k, n}^{(2)}=\sum_{i=0}^{N_{t}-1} H_{k, i} a_{k, n-2 i}+v_{k, n} .
$$




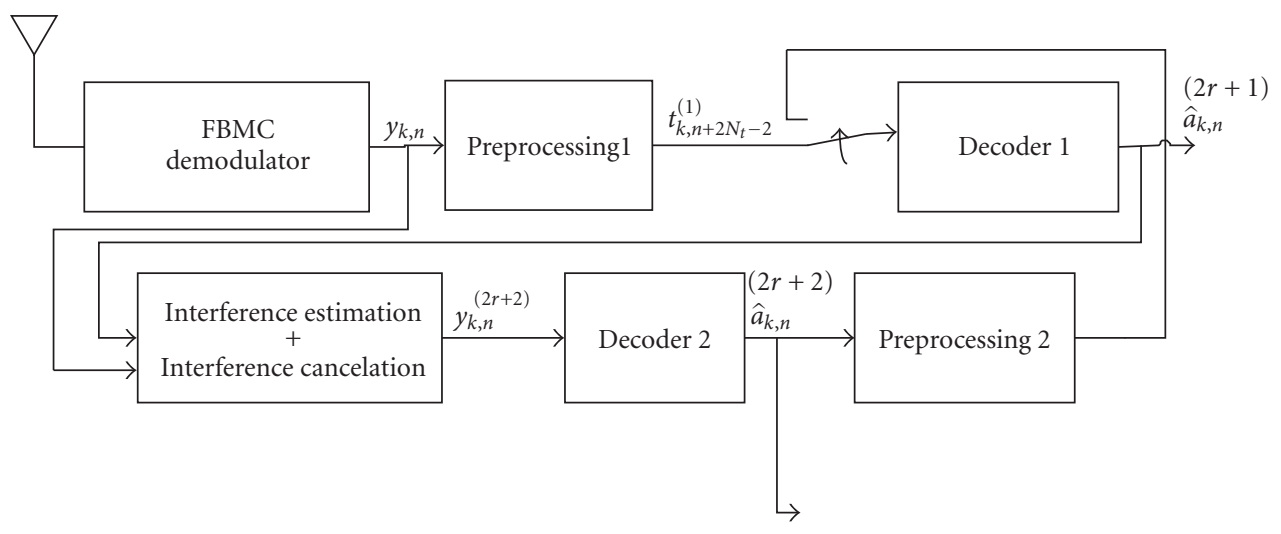

FIGURE 3: Receiver decoding processing for FBMC modulation in the case of single delay STTC transmission.

The operation of estimating $u_{k, n}$ and canceling its contribution to the signal $y_{k, n}$ is referred to as "Interference estimation + Interference cancelation" as depicted in Figure 3. Thus, we can perform from $y_{k, n}^{(2)}$ a new decoding (Decoder 2 block) to obtain a new estimate $\hat{a}_{k, n}^{(2)}$ of $a_{k, n}$. In the same manner, we can use either a Viterbi/ML decoding or a linear decoder. From $\hat{a}_{k, n}^{(2)}$ and (19) we can also compute $t_{k, n+2}^{(2)}$ by

$$
t_{k, n+2 N_{t}-2}^{(2)}=\sum_{q=1}^{N_{t}-1} \gamma_{q} \hat{a}_{k, n-2 q}^{(2)}+\mu_{k} \hat{a}_{k, n}^{(2)}+\sum_{q=1}^{N_{t}-1} \beta_{q} \hat{a}_{k, n+2 q}^{(2)} .
$$

$t_{k, n+2}^{(2)}$ can also be rewritten as

$$
\begin{aligned}
t_{k, n+2 N_{t}-2}^{(2)}= & \sum_{q=1}^{N_{t}-1} \gamma_{q} a_{k, n-2 q}+\mu_{k} a_{k, n}+\sum_{q=1}^{N_{t}-1} \beta_{q} a_{k, n+2 q} \\
& +\sum_{q=1}^{N_{t}-1} \gamma_{q}\left(\hat{a}_{k, n-2 q}^{(2)}-a_{k, n-2 q}\right)+\mu_{k}\left(\hat{a}_{k, n}^{(2)}-a_{k, n}\right) \\
& +\sum_{q=1}^{N_{t}-1} \beta_{q}\left(\hat{a}_{k, n+2 q}^{(2)}-a_{k, n+2 q}\right) \\
= & \sum_{q=1}^{N_{t}-1} \gamma_{q} a_{k, n-2 q}+\mu_{k} a_{k, n}+\sum_{q=1}^{N_{t}-1} \beta_{q} a_{k, n+2 q} \\
& + \text { noise component. }
\end{aligned}
$$

$t_{k, n+2 N_{t}-2}^{(2)}$ is a new version of the $t_{k, n+2 N_{t}-2}^{(1)}$ signal which is obtained from the estimates of the Decoder 2 block output. Thus, this last equation can be used to perform another estimation $\hat{a}_{k, n}^{(3)}$ of $a_{k, n}$ in the same manner as we compute $\hat{a}_{k, n}^{(1)}$. We expect to improve the estimation of $a_{k, n}$ since the noise component in (30) should be less correlated than the one in (19). Again from $\hat{a}_{k, n}^{(3)}$ we can derive an estimate $\hat{u}_{k, n}^{(2)}$ of $u_{k, n}$ as in (25). Therefore, we can repeat another decoding process as already presented. We can run this decoding process as many times as necessary. The process of computing $t_{k, n+2 N_{t}-2}^{(2)}$ from the $\hat{a}_{k, n}^{(2)}$ is referred to as Preprocessing2; see Figure 3. Let us have a look at the convergence of this iterative method.

5.2. A Convergence Analysis of the Iterative Procedure. Let us consider the function $P_{e}=C_{1}(\mathrm{SNR})$ that we obtain when considering the perfect cancelation of the interference term by using (28) and the function $P_{e}=C_{2}$ (SNR) obtained using (19). $P_{e}$ is the real symbol error probability and SNR = $2 \sigma_{a}^{2} / N_{0}=1 / N_{0}$ assuming that the real symbol power $\sigma_{a}^{2}$ is fixed at 1/2. These functions are illustrated in Figure 4 for a given channel realization. Let us note that $C_{1}$ is $\Delta \mathrm{dB}$ better than $C_{2}$, that is,

$$
C_{1}\left(\frac{1}{\left(1+\alpha_{\Delta}\right) N_{0}}\right)=C_{2}\left(\frac{1}{N_{0}}\right),
$$

with $\Delta=10 \log _{10}\left(1+\alpha_{\Delta}\right)$. At the first iteration, when using (19) for decoding, we obtain at $\mathrm{SNR}=1 / N_{0}$ a symbol probability of error $P_{e 1}=C_{2}\left(1 / N_{0}\right)$. This first iteration is summarized by the point $A_{1}\left(1 / N_{0}, P_{e 1}\right)$ in Figure 4 . Now, from this probability of error we can derive the degradation that we obtain when applying interference cancelation. Indeed, the cancelation of the interference will add some noise to the current noise component. This additional noise component is given by the cancelation error

$$
\begin{aligned}
n^{+}= & \sum_{i=0}^{N_{t}-1} j H_{k, i}\left(u_{k, n-2 i}-\hat{u}_{k, n-2 i}^{(1)}\right) \\
= & \sum_{i=0}^{N_{t}-1} j H_{k, i} \sum_{(p, q) \neq(0,0)}\left(a_{k+p, n-2 i+q}-\hat{a}_{k+p, n-2 i+q}^{(1)}\right) \\
& \times \sum_{m=-\infty}^{\infty} g_{k, n-2 i}[m] g_{k+p, n-2 i+q}[m] .
\end{aligned}
$$

Using the current observation

$$
\begin{array}{ll}
\hat{a}_{k, n}=a_{k, n} & \text { with probability } 1-P_{e 1}, \\
\hat{a}_{k, n} \neq a_{k, n} & \text { with probability } P_{e 1}
\end{array}
$$


and considering that $[10]$

$$
\sum_{(p, q) \neq(0,0)}\left|\sum_{m=-\infty}^{\infty} g_{k, n-2 i}[m] g_{k+p, n-2 i+q}[m]\right|^{2}=1,
$$

we have

$$
E\left\{\left|n^{+}\right|^{2}\right\}=P_{e 1} \underbrace{\sum_{i=0}^{N_{t}-1}\left|H_{k, i}\right|^{2}}_{\alpha_{h}} .
$$

Therefore, the symbol probability of error is given at second iteration by

$$
\begin{aligned}
P_{e 2} & =C_{1}\left(\frac{1}{N_{0}^{1}}\right)=C_{1}\left(\frac{1}{N_{0}+P_{e 1} \alpha_{h}}\right) \\
& =C_{1}\left(\frac{1}{N_{0}\left(1+\alpha_{h} C_{2}\left(1 / N_{0}\right) / N_{0}\right)}\right),
\end{aligned}
$$

where $1 / N_{0}^{1}$ is the SNR at the input of Decoder 2 .

$C_{2}\left(1 / N_{0}\right)$ is a $Q$-function that is exponentially decreasing as SNR increases; thus, $\alpha_{h} C_{2}\left(1 / N_{0}\right) / N_{0}$ decreases as SNR increases since the exponential function overwhelms the polynomial function. Then, there is a noise power $N_{0}^{a}$ such that, for $N_{0}<N_{0}^{a}$

$$
\alpha_{h} \frac{C_{2}\left(1 / N_{0}\right)}{N_{0}}<\alpha_{\Delta}
$$

and thus,

$$
\frac{1}{N_{0}\left(1+\alpha_{h} C_{2}\left(1 / N_{0}\right) / N_{0}\right)}>\frac{1}{\left(1+\alpha_{\Delta}\right) N_{0}} .
$$

Therefore for $N_{0}<N_{0}^{a}$,

$$
\begin{aligned}
& C_{1}\left(\frac{1}{N_{0}\left(1+\alpha_{h} C_{2}\left(1 / N_{0}\right) / N_{0}\right)}\right) \\
& \quad<C_{1}\left(\frac{1}{\left(1+\alpha_{\Delta}\right) N_{0}}\right)=C_{2}\left(\frac{1}{N_{0}}\right),
\end{aligned}
$$

that is,

$$
P_{e 2}<P_{e 1}
$$

For $N_{0}<N_{0}^{a}$ the output of the second iteration will give better performance than that of the first iteration. This second iteration is summarized by the point $A_{2}\left(1 / N_{0}^{1}, P_{e 2}\right)$ in Figure 4.

When recombining the signal at the input of Decoder 1 for the third iteration using (29), the noise component is now smaller than that in the previous case since $P_{e 2}<P_{e 1}$.

Consequently, the third iteration performance is given by $C_{2}$ at $\mathrm{SNR}=1 / N_{0}^{2}$ with $N_{0}^{2}<N_{0}^{1}$. Thus, $C_{2}\left(1 / N_{0}^{2}\right)<$ $C_{2}\left(1 / N_{0}\right)$, that is, the probability of error at the output of Decoder 1 for the third iteration $P_{e 3}$ is less than that for $P_{e 1}$. This third iteration is summarized by the point $A_{3}\left(1 / N_{0}^{2}, P_{e 3}\right)$ in Figure 4. Let us notice that $P_{e 3}$ could be greater than $P_{e 2}$.

The next iteration performance can be derived in the same manner since we just have to replace $N_{0}$ by $N_{0}^{2}$. Thus, the probability of error at the output of a given decoder (Decoder 1 or Decoder 2) will always decrease or reach a fixed point.

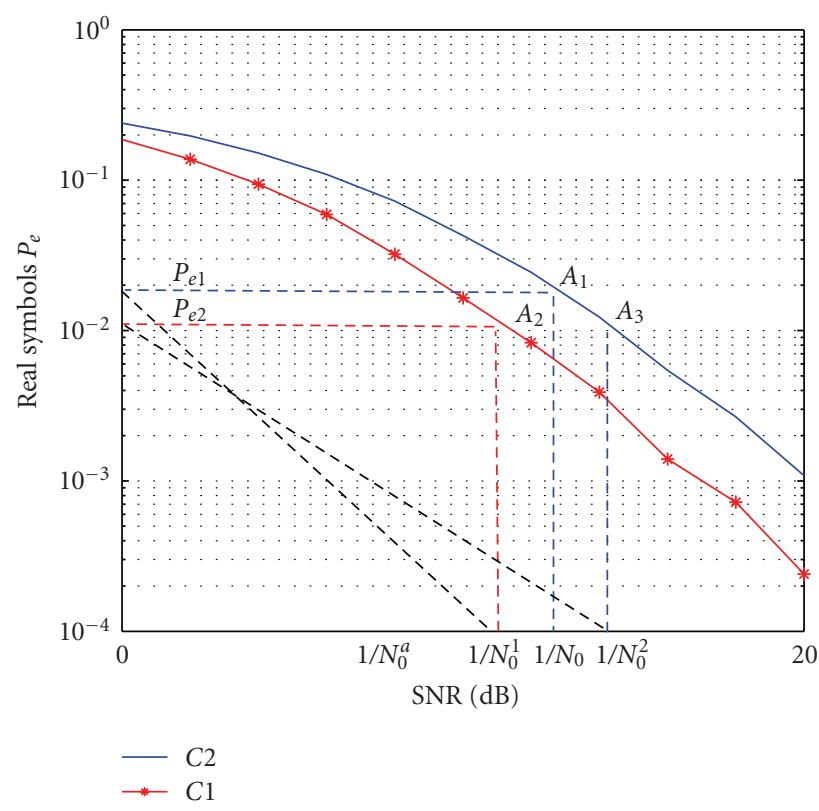

Figure 4: Convergence illustration.

\section{Simulation Results}

In this section, we will evaluate the performance of the two decoding methods that we have presented. We consider a transmission scheme with two and three transmit antennas.

For $N_{t}=2$, we have

$$
\begin{aligned}
t_{k, n+2 N_{t}-2}^{(1)}= & \left|H_{k, 1}\right|^{2} a_{k, n-2}+2 \Re\left\{H_{k, 1}^{*} H_{k, 0}\right\} a_{k, n} \\
& +\left|H_{k, 0}\right|^{2} a_{k, n+2}+w_{k, n+2 N_{t}-2}
\end{aligned}
$$

and for $N_{t}=3$, we get

$$
\begin{aligned}
t_{k, n+2 N_{t}-2}^{(1)}= & \left|H_{k, 2}\right|^{2} a_{k, n-4}+2 \mathfrak{R}\left\{H_{k, 2}^{*} H_{k, 1}\right\} a_{k, n-2} \\
& +\left(2 \Re\left\{H_{k, 2}^{*} H_{k, 0}\right\}+\left|H_{k, 1}\right|^{2}\right) a_{k, n} \\
& +2 \Re\left\{H_{k, 1}^{*} H_{k, 0}\right\} a_{k, n+2}+\left|H_{k, 0}\right|^{2} a_{k, n+4} .
\end{aligned}
$$

The simulation parameters we consider are given as follows:

(i) no channel coding,

(ii) QPSK modulation,

(iii) Rayleigh channel per antenna, that is, flat over all the subcarriers. We assume that the channel coefficients are perfectly known by the receiver,

(iv) number of subcarrier $M=32$,

(v) we used a truncation of the IOTA (Isotropic Orthogonal Transform Algorithm) prototype function [1]. Its duration is limited to $4 T_{0}$, which leads to a nearly orthogonal prototype filter containing $L=4 M=128$ taps. 


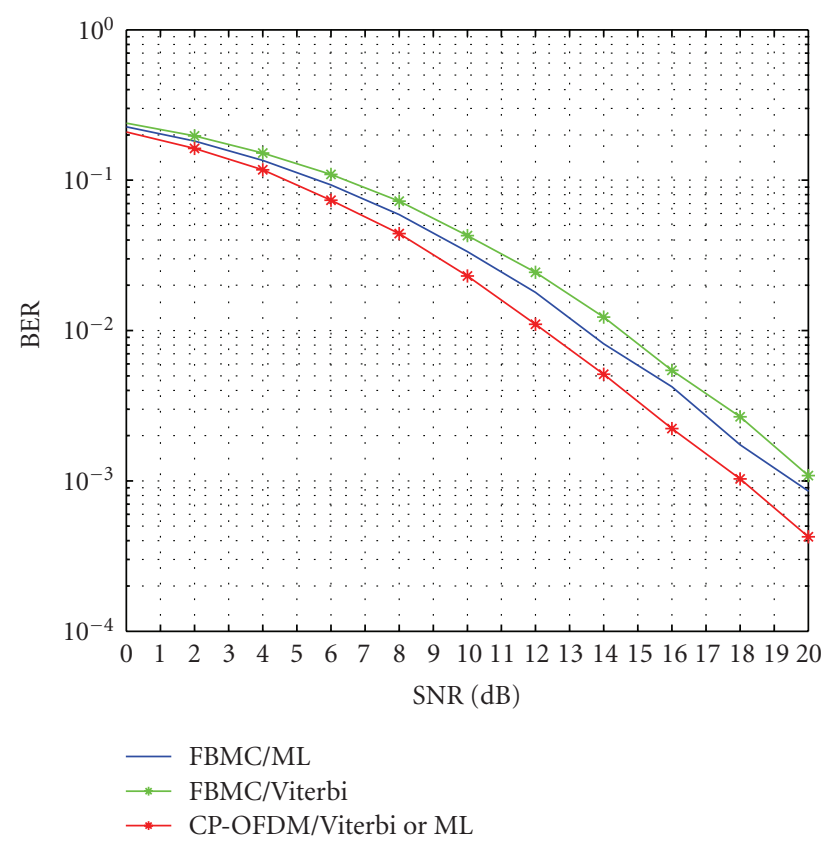

FIGURE 5: Performance of single delay STTC with 2 transmit antennas and one receive antenna (FBMC and CP-OFDM modulation).

In this section, we give BER (Bit Error Rate) versus SNR simulation results, and consequently, we do not take into consideration the loss of efficiency due to the cyclic prefix in CP-OFDM modulation.

In Figure 5 we show the performance of the FBMC decoding structure introduced in Figure 2. For FBMC, we consider both ML and Viterbi decoding. ML decoding using an exhaustive search among all possible transmitted sequences of data outperforms Viterbi decoding by $1 \mathrm{~dB}$. This is due to the fact that the noise is colored; thus, Viterbi decoding is suboptimal. We also give the CP-OFDM performance using a Viterbi decoding. We can see that CPOFDM outperforms ML/FBMC by about $1 \mathrm{~dB}$.

In the rest of this section, we will focus on the iterative decoding performance. The simulation results are obtained using Viterbi decoding blocks implemented inside Decoder 1 and Decoder 2 blocks in Figure 3. The Viterbi algorithm implemented in Decoder 1 is related to (41). For QPSK modulation, the Trellis is a $4^{N_{T}-1}$ state Trellis with only two possible transitions per state since the detection is performed on real data. Whereas the Viterbi algorithm implemented in Decoder 2 is related to $(28)$ and is a $2^{N_{T}-1}$ state Trellis with two transitions per state, again detection is performed on real data. We also consider hard estimation of the data at the output of a given Viterbi decoder. For the CP-OFDM case with QPSK modulation, we have a $4^{N_{T}-1}$ state Trellis with 4 transitions per state as the detection is performed on complex data. Therefore, this Viterbi algorithm is more complex compared with one of the two Viterbi algorithms used in the case of FBMC modulation. The two Viterbi algorithms used in FBMC taken together have a complexity comparable to the one used with CP-OFDM. However, the two Viterbi algorithms used in FBMC operate on a

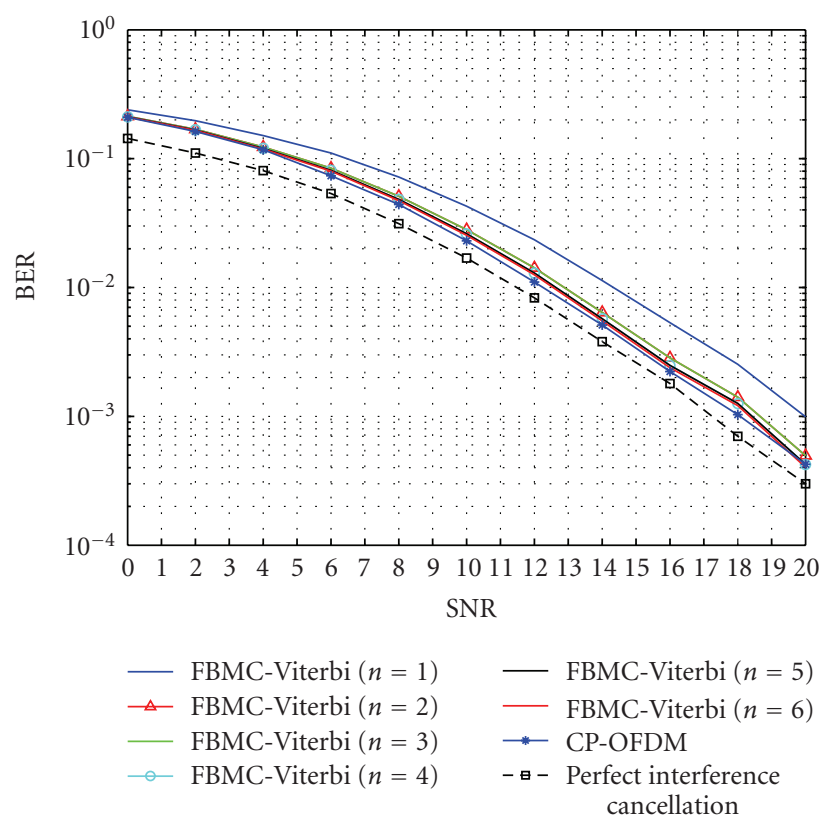

FIGURE 6: Performance of single delay STTC (iterative decoding) with 2 transmit antennas and one receive antenna (FBMC and CPOFDM modulation).

frame sequence which is two times longer than the one for CP-OFDM modulation. Then, in terms of complexity the proposed FBMC structure has a significantly higher complexity than that of CP-OFDM mainly due to the "Interference estimation + Interference cancelation" block.

For uncorrelated Rayleigh channels, we plot the performance of this FBMC receiver structure for different iteration stages as well as the performance of CP-OFDM with ML decoding as a matter of comparison. Figures 6 and 7 provide the simulation results for $N_{t}=2$ and $N_{t}=3$, respectively. For $n=1$, we have a $2 \mathrm{~dB}$ degradation compared to CPOFDM. For $n \geq 2$ (more than two-Viterbi decoding), we get closer to CP-OFDM. For $n=5$ or 6 , we almost reach the same performance as that of CP-OFDM. In Figure 6 we also plot the curve obtained when we assume perfect interference cancelation in the second iteration as mentioned in (28). In that case, there is a possible gain of $0.8 \mathrm{~dB}$ since the Viterbi structure with 2 states and two transitions per state (Decoder 2) provides better performance than the 4-state Viterbi decoder with 4 transitions per state implemented for CP-OFDM. Indeed, it is possible to show that the structures of the code related to these two Trellises have the same minimum distance. However, the performance gain is due to the distance distribution associated to the two Trellises.

Moreover, let us evaluate this scheme in presence of a frequency selective channel. We consider the following channel parameters:

(i) uncoded QPSK modulation,

(ii) $M=64$ subcarriers,

(iii) static channels (no Doppler), IOTA prototype, 


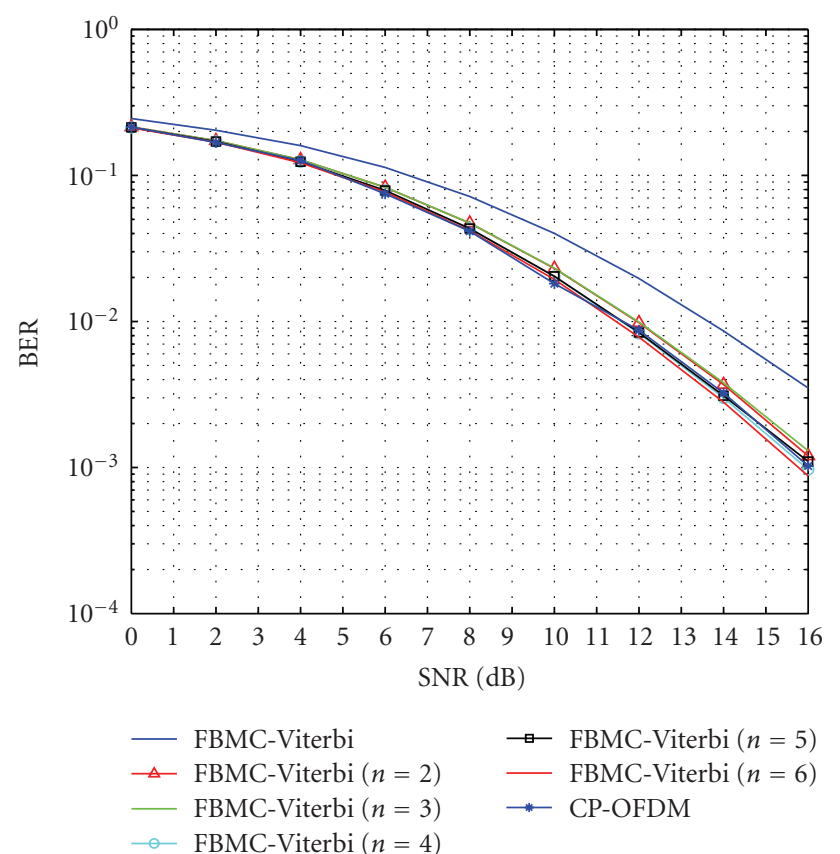

FIGURE 7: Performance of single delay STTC (iterative decoding) with 3 transmit antennas and one receive antenna (FBMC and CPOFDM modulation).

(iv) 3-tap channels between the transmit antennas and the receive: power profile: $0,-4,-10(\mathrm{~dB})$ Delay: 0 , 1, 2 (number of samples),

(v) OFDM Cyclic Prefix length: 4 samples,

(vi) perfect channel estimation.

As shown in Figure 8, after one iteration $(n=1)$, we have about $2 \mathrm{~dB}$ degradation compared to CP-OFDM. For $n=2$ and $n=3$, the loss is reduced to $0.7 \mathrm{~dB}$, and for $n=6$ the degradation is about $0.3 \mathrm{~dB}$ compared to CPOFDM. However, the iterative method has an inherent gain as FBMC does not use a CP contrary to CP-OFDM.

\section{Conclusion}

In this paper, we have presented two general methods for data detection when combining FBMC and single delay STTC as well as the interference cancelation and the iterative methods. The interference cancelation method despite its simplicity has poorer performance compared to that of CPOFDM. Thus, we have proposed an iterative decoding based on interference estimation and cancelation which does not require any channel coding or decoding block. We have shown that in the case of QPSK modulation and Rayleigh or frequency selective channels it is possible with this decoding method to perform as better as OFDM-STTC. Moreover if the iterative cancelation process is improved, then a potential gain can be achieved. This is obtained with a relatively higher complexity. In future work, we will look at FBMC with other STTC schemes and evaluate their performance under nonlocally flat channels.

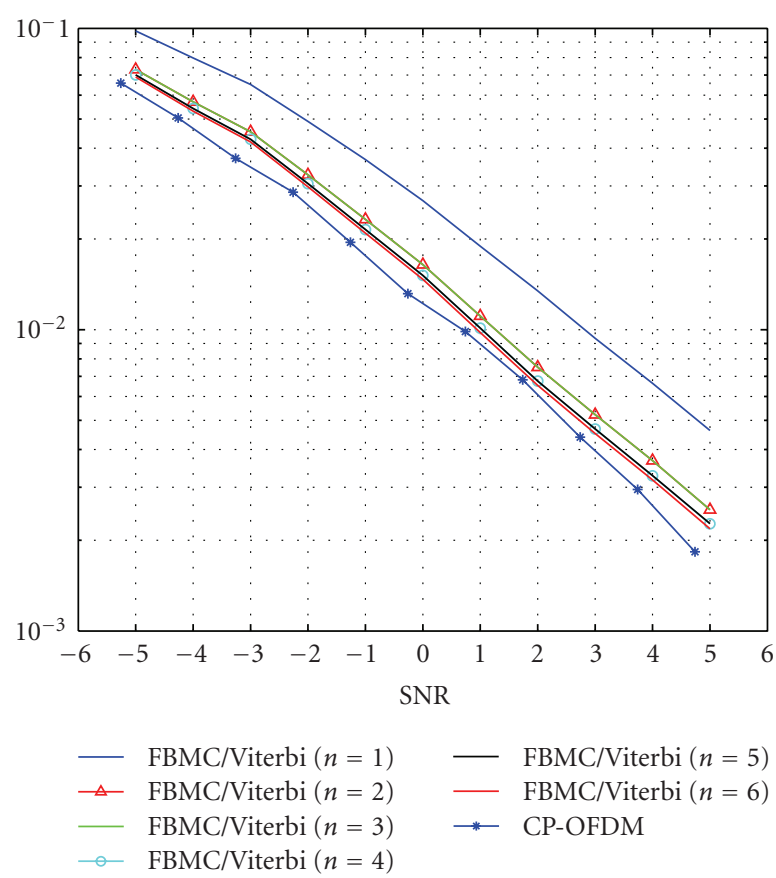

FIGURE 8: Performance of single delay STTC (iterative decoding) with 2 transmit antennas and one receive antenna over frequency selective channels.

\section{Appendix}

\section{A. General Expression of $A_{k, n}, B_{k, n}$, and $C_{k, n}$}

A.1. $A_{k, n}$ Value. Let us compute $A_{k, n}$.

(i) Case $N_{t}$ is even, that is, $N_{t}=2 U_{t}$,

$$
A_{k, n}=x_{k, n}\left(\sum_{i=0}^{U_{t}-1} H_{k, 2 U_{t}-1-i}^{*} H_{k, i}+\sum_{i=U_{t}}^{2 U_{t}-1} H_{k, 2 U_{t}-1-i}^{*} H_{k, i}\right)
$$

Using the relation $q=2 U_{t}-1-i$, we have

$$
\begin{aligned}
A_{k, n} & =x_{k, n}\left(\sum_{i=0}^{U_{t}-1} H_{k, 2 U_{t}-1-i}^{*} H_{k, i}+\sum_{q=0}^{U_{t}-1} H_{k, q}^{*} H_{k, 2 U_{t}-1-q}\right) \\
& =x_{k, n}\left(\sum_{i=0}^{U_{t}-1}\left(H_{k, 2 U_{t}-1-i}^{*} H_{k, i}+H_{k, i}^{*} H_{k, 2 U_{t}-1-i}\right)\right) \\
& =x_{k, n} \underbrace{\left(2 \sum_{i=0}^{U_{t}-1} \Re\left\{\left(H_{k, 2 U_{t}-1-i}^{*} H_{k, i}\right)\right\}\right)}_{\mu_{k}} .
\end{aligned}
$$

(ii) Case $N_{t}$ is odd, that is, $N_{t}=2 U_{t}+1$,

$$
\mu_{k}=\left(\sum_{i=0}^{U_{t}-1} H_{k, 2 U_{t}-i}^{*} H_{k, i}+H_{k, U_{t}}^{*} H_{k, U_{t}}+\sum_{i=U_{t}+1}^{2 U_{t}} H_{k, 2 U_{t}-i}^{*} H_{k, i}\right)
$$


Again using $q=2 U_{t}-i$, we have

$$
\mu_{k}=\left(2 \sum_{i=0}^{U_{t}-1} \mathfrak{R}\left\{\left(H_{k, 2 U_{t}-i}^{*} H_{k, i}\right)\right\}+\left|H_{k, U_{t}}\right|^{2}\right),
$$

and we get

$$
A_{k, n}=x_{k, n} \mu_{k}
$$

A.2. $B_{k, n}$ Value. Let us now compute $B_{k, n}$; setting $q=p-i$, we get

$$
\begin{aligned}
B_{k, n} & =\sum_{i=1}^{N_{t}-1} \sum_{p=0}^{i-1} H_{k, N_{t}-1-p}^{*} H_{k, i} x_{k, n+2 p-2 i} \\
& =\sum_{i=1}^{N_{t}-1} \sum_{q=1}^{i} x_{k, n-2 q} H_{k, N_{t}-1+q-i}^{*} H_{k, i} .
\end{aligned}
$$

This last equation is the sum over a triangular set of index; therefore, the sum can be taken either from lines or from columns where the total is the same. Therefore,

$$
B_{k, n}=\sum_{q=1}^{N_{t}-1} x_{k, n-2 q} \sum_{i=q}^{N_{t}-1} H_{k, N_{t}-1+q-i}^{*} H_{k, i} .
$$

Taking $m=i-q$, we get

$$
B_{k, n}=\sum_{q=1}^{N_{t}-1} x_{k, n-2 q} \underbrace{\sum_{m=0}^{N_{t}-1-q} H_{k, N_{t}-1-m}^{*} H_{k, m+q}}_{\gamma_{q}} .
$$

(i) Case $N_{t}-q$ is even, that is, $N_{t}-q=2 U_{q}$; then,

$$
\begin{aligned}
\gamma_{q}= & \sum_{m=0}^{N_{t}-1-q} H_{k, N_{t}-1-m}^{*} H_{k, m+q} \\
= & \sum_{m=0}^{U_{q}-1} H_{k, 2 U_{q}+q-1-m}^{*} H_{k, m+q} \\
& +\sum_{m=U_{q}}^{2 U_{q}-1} H_{k, 2 U_{q}+q-1-m}^{*} H_{k, m+q} \\
= & 2 \sum_{m=0}^{U_{q}-1} \Re\left\{H_{k, 2 U_{q}+q-1-m}^{*} H_{k, m+q}\right\} .
\end{aligned}
$$

(ii) Case $N_{t}-q$ is odd, that is, $N_{t}-q=2 U_{q}+1$; then,

$$
\begin{aligned}
\gamma_{q}= & \sum_{m=0}^{N_{t}-1-q} H_{k, N_{t}-1-m}^{*} H_{k, m+q} \\
= & \sum_{m=0}^{U_{q}-1} H_{k, 2 U_{q}+q-m}^{*} H_{k, m+q}+H_{k, U_{q}+q}^{*} H_{k, U_{q}+q} \\
& +\sum_{m=U_{q}+1}^{2 U_{q}} H_{k, 2 U_{q}+q-m}^{*} H_{k, m+q} \\
= & 2 \sum_{m=0}^{U_{q}-1} \Re\left\{H_{k, 2 U_{q}+q-m}^{*} H_{k, m+q}\right\}+\left|H_{k, U_{q}+q}^{*}\right|^{2} .
\end{aligned}
$$

A.3. $C_{k, n}$ Value. Let us now compute $C_{k, n}$; setting $q=p-i$, we get

$$
C_{k, n}=\sum_{i=1}^{N_{t}-2} \sum_{q=1}^{N_{t}-1-i} H_{k, N_{t}-1-q-i}^{*} H_{k, i} x_{k, n+2 q} .
$$

This last equation is the sum over a triangular set of index; therefore, the sum can be taken either from lines or from columns where the total is the same. Therefore,

$$
C_{k, n}=\sum_{q=1}^{N_{t}-1} x_{k, n+2 q} \underbrace{\sum_{i=0}^{N_{t}-1-q} H_{k, N_{t}-1-q-i}^{*} H_{k, i}}_{\beta_{q}} .
$$

(i) Case $N_{t}-q$ is even, that is, $N_{t}-q=2 U_{q}$; then,

$$
\begin{aligned}
\beta_{q} & =\sum_{i=0}^{N_{t}-1-q} H_{k, N_{t}-1-q-i}^{*} H_{k, i} \\
& =\sum_{i=0}^{U_{q}-1} H_{k, 2 U_{q}-1-i}^{*} H_{k, i}+\sum_{m=U_{q}}^{2 U_{q}-1} H_{k, 2 U_{q}-1-i}^{*} H_{k, i} \\
& =2 \sum_{i=0}^{U_{q}-1} \Re\left\{H_{k, 2 U_{q}-1-i}^{*} H_{k, i}\right\} .
\end{aligned}
$$

(ii) Case $N_{t}-q$ is odd, that is, $N_{t}-q=2 U_{q}+1$; then,

$$
\begin{aligned}
\beta_{q}= & \sum_{i=0}^{N_{t}-1-q} H_{k, N_{t}-1-q-i}^{*} H_{k, i}=\sum_{i=0}^{U_{q}-1} H_{k, 2 U_{q}-i}^{*} H_{k, i}+H_{k, U_{q}}^{*} H_{k, U_{q}} \\
& +\sum_{m=U_{q}+1}^{2 U_{q}} H_{k, 2 U_{q}-i}^{*} H_{k, i} \\
= & 2 \sum_{m=0}^{U_{q}-1} \Re\left\{H_{k, 2 U_{q}-i}^{*} H_{k, i}\right\}+\left|H_{k, U_{q}}^{*}\right|^{2} \cdot
\end{aligned}
$$

\section{Acknowledgments}

The authors would like to thank Pr. M. Bellanger for helpful discussions. This work was supported in part by the European Commission under Project PHYDYAS (FP7-ICT2007-1-211887).

\section{References}

[1] B. Le Floch, M. Alard, and C. Berrou, "Coded orthogonal frequency division multiplex," Proceedings of the IEEE, vol. 83, pp. 982-996, 1995.

[2] H. Boelcskei, "Orthogonal frequency division multiplexing based on offset QAM," in Advances in Gabor Analysis, Birkhäuser, Boston, Mass, USA, 2003.

[3] P. Siohan, C. Siclet, and N. Lacaille, "Analysis and design of OFDM/OQAM systems based on filterbank theory," IEEE Transactions on Signal Processing, vol. 50, no. 5, pp. 1170-1183, 2002. 
[4] R. W. Chang, "Synthesis of band-limited orthogonal signals for multi-channel data transmission," Bell Labs Technical Journal, vol. 45, pp. 1775-1796, 1966.

[5] B. R. Saltzberg, "Performance of an efficient parallel data transmission system," IEEE Transactions on Communication Technology, vol. 15, no. 6, pp. 805-811, 1967.

[6] V. Tarokh, N. Seshadri, and A. R. Calderbank, "Space-time codes for high data rate wireless communication: performance criterion and code construction," IEEE Transactions on Information Theory, vol. 44, no. 2, pp. 744-765, 1998.

[7] V. Tarokh, H. Jafarkhani, and A. R. Calderbank, "Spacetime block coding for wireless communications: performance results," IEEE Journal on Selected Areas in Communications, vol. 17, no. 3, pp. 451-460, 1999.

[8] M. Bellanger, "Transmit diversity in multicarrier transmission using OQAM modulation," in Proceedings of the 3rd International Symposium on Wireless Pervasive Computing (ISWPC '08), pp. 727-730, Santorini, Greece, May 2008.

[9] B. Hirosaki, "Orthogonally multiplexed QAM system using the discrete Fourier transform," IEEE Transactions on Communications Systems, vol. 29, no. 7, pp. 982-989, 1981.

[10] C. Lélé, P. Siohan, R. Legouable, and J.-P. Javaudin, "Preamblebased channel estimation techniques for OFDM/OQAM over the powerline," in Proceedings of IEEE International Symposium on Power Line Communications and Its Applications (ISPLC '07), pp. 59-64, Pisa, Italy, March 2007.

[11] G. D. Forney Jr., "Maximum-likelihood sequence estimation of digital sequences in the presence of intersymbol interference," IEEE Transactions on Information Theory, vol. 18, no. 3, pp. 363-378, 1972.

[12] J.-D. Wang and H. Y. Chung, "Trellis coded communication systems in the presence of colored noise: performance analysis, simulation, and the swapping technique," in Proceeingds of IEEE Conference Record on Global Telecommunications Conference, and Exhibition. 'Communications for the Information Age' (GLOBECOM '88), vol. 2, pp. 1160-1165, Hollywood, Fla, USA, November-December 1988. 